01

\title{
Пики термодесорбции водорода: моделирование и интерпретация
}

\author{
(С Ю.В. Заика, ${ }^{1}$ Е.К. Костикова, ${ }^{1}$ Ю.С. Нечаев ${ }^{2}$ \\ ${ }^{1}$ Институт прикладных математических исследований КарНЦ РАН, \\ 185910 Петрозаводск, Россия \\ ${ }^{2}$ Центральный научно-исследовательский институт черной металлургии им. И.П. Бардина, \\ 105005 Москва, Россия \\ e-mail: zaika@krc.karelia.ru
}

Поступило в Редакцию 19 июня 2020 г.

В окончательной редакции 20 августа 2020 г.

Принято к публикации 25 августа 2020 г.

\begin{abstract}
Проанализированы различные модели пиков термодесорбции водорода. Модель динамики усредненной по объему концентрации с континуальным параметром позволила учесть интегрально степень доминирования лимитирующих факторов диффузии и рекомбинации атомов в молекулы при десорбции. Предложен аналитический критерий симметричности пиков в контексте сравнения с методикой разложения составного спектра на сумму гауссианов. Предложены модификации метода Киссинджера для оценки энергии активации десорбции в экспериментах с несколькими скоростями нагрева материала и процедуры решения обратной задачи параметрической идентификации унимодального пика лишь по одной скорости нагрева. Проведен сравнительный анализ с диффузионной моделью с динамическими граничными условиями. Показано, что причиной локальных пиков может быть не только высвобождение водорода, захваченного в объеме с различными энергиями связи, но и динамика взаимодействия объемных и поверхностных процессов, изменение структуры поверхности в процессе нагрева. Оценены параметры термодесорбции дейтерия из изотропного графита ISO-880U.
\end{abstract}

Ключевые слова: термодесорбция водорода, моделирование ТДС пиков, параметрическая идентификация.

DOI: $10.21883 /$ JTF.2021.02.50355.208-20

\section{Введение}

Интерес к взаимодействию изотопов водорода с твердым телом носит многоплановый характер [1-5]: защита конструкционных материалов от водородной коррозии, транспортировка углеводородов, водородная энергетика, перспективы термоядерного синтеза. Одним из наиболее информативных методов исследования кинетики взаимодействия новых материалов с водородом является термодесорбционная спектрометрия (ТДС). Укажем лишь некоторые работы, близкие к излагаемому материалу и содержащие в совокупности обширный список литературы по данной тематике.

Общий анализ и практические рекомендации содержатся в обзорах [6,7]. Подробно изложены разработки методики исследования спектров на основе классического метода Киссинджера. Соответствующие модели оперируют усредненной по объему образца концентрацией абсорбированного водорода. Отметим обзор моделей в работе [8] и анализ границ применимости формулы Киссинджера в сопоставлении с распределенной моделью с обратимым захватом диффузанта ловушками (модель МакНабба-Фостера) [9]. Эффекту захвата в ТДС экспериментах посвящена работа [10]. В работах [11-13] представлены исследования в области водородного материаловедения для термоядерной энергетики. Особое место занимает гидридная тематика. Общая распределенная модель дегидрирования изложена в [14], прикладные задачи и соответствующие модели представле- ны в [15-17]. Математические модели термодесорбции и водородопроницаемости с учетом различных стадий переноса и численные методы решения краевых задач описаны в [18-20].

Работа [21] акцентирована на проблемах интерпретации спектров. Трудности их анализа типичны для некорректных обратных задач: неединственность решения, неоднозначность интерпретации и высокая чувствительность к погрешностям входных данных. Типичными являются нулевые граничные условия для объемной концентрации в условиях вакуумирования. Техническая причина понятна, поскольку математические пакеты не содержат для диффузионных уравнений динамических граничных условий (с производной по времени); тем самым исключается из рассмотрения динамика поверхностных процессов. В наблюдаемом многопиковом спектре приходится объяснять локальные пики влиянием захвата в объеме с различными энергиями связи.

Численные эксперименты показывают [18,20], что, как минимум, два ярко выраженных пика может дать динамика взаимодействия „поверхность-объем“ (при стандартном уравнении диффузии в объеме без слагаемых обратимого захвата). В [20] приведены (рис. 10) графики, когда в рамках модели с уравнением диффузии описываются сразу два пика для экспериментальных данных (сталь, бериллий, вольфрам). Схематично:

1) десорбция с поверхности и подповерхностного слоя дает локальный пик потока и обедняет водородом подповерхностный слой, что и влечет локальный спад потока; 
2) с дальнейшим нагревом интенсифицируется диффузия, реагируя на большой градиент концентрации вблизи поверхности;

3 ) подкачка из объема атомарного водорода порождает дополнительный всплеск (при недостаточной активности десорбции концентрация на поверхности некоторое время может даже возрастать!).

Подчеркнем, что такая ситуация численно наблюдается, если энергии активации диффузии и десорбции находятся в диапазонах, когда ни один из факторов не является единственным лимитирующим. Локальные пики не обязательно связывать с различными энергиями связи атомов водорода в объеме материала. Это важно, поскольку экспериментальные результаты на исчезающе тонких мембранах экстраполируются на защитные конструкции реакторов, включая проект ITER. Ловушки (захват неоднородностями материала), безусловно, существуют. Но определяющее влияние захвата в объеме, по существу прикладной задачи, необходимо обосновывать.

Настоящая работа содержит результаты применительно к экспериментальным данным и теоретическим положениям в работах $[2,22]$. Более подробный анализ экспериментальных данных по углеродным наноструктурам и графиту проведен в [23].

\section{1. Модель в форме реакции порядка $\alpha \in[1,2]$}

Рассмотрим вначале уравнение реакции первого порядка $\dot{X}(t)=-K(T) X(t)$. Здесь $X(t) \quad$ - текущая усредненная концентрация водорода в образце, $K(T)=K_{0} \exp \{-Q / R T\} \quad$ (аррениусовская зависимость кинетического коэффициента от температуры), $T(t)=T_{0}+\beta t$ (равномерный линейный нагрев с невысокой скоростью $\beta,[\beta]=\mathrm{K} / \mathrm{s})$.

Чтобы пояснить мотивацию, приведем кратко схему вывода уравнения, когда дегазация лимитирована диффузией. Рассмотрим простейшую краевую задачу $c_{t}=D(T(t)) c_{x x} \quad[t>0, x \in(0, h)], \quad c=c_{0}>0$ $[t=0], c=0[t>0, x=0, h]$. Здесь $c-$ концентрация $\left([c]=1 \mathrm{H} / \mathrm{cm}^{3}\right), D-$ коэффициент диффузии, $h-$ толщина пластины. В начальный момент времени атомарный водород распределен равномерно. Затем, вследствие вакуумирования мгновенно (в относительном масштабе времени) устанавливаются нулевые концентрации в подповерхностном объеме пластины.

После перехода к безразмерным переменным $\tilde{t}=\int_{0}^{t} D(T(s)) d s / h^{2}, \quad \tilde{x}=x / h, \quad \tilde{c}=c / c_{0} \quad$ получаем формально $D=1, \quad h=1, \quad c_{0}=1$ (в выкладках в пределах абзаца оставляем прежние обозначения, не усложняя их тильдами). Тогда для усредненной переменной $\bar{c}(t)=\int_{0}^{1} c(t, x) d x$ (см., например, справедливо представление

$$
\bar{c}(t)=\sum_{n=1,3,5 \ldots} \frac{8}{\pi^{2} n^{2}} \exp \left(-\frac{n^{2} t}{\tau}\right), \tau \equiv \frac{1}{\pi^{2}} .
$$

При анализе ТДС спектров нас интересует производная $d \bar{c} / d t$, которая при $t=0$ не определяется почленным дифференцированием ряда. Это следствие несогласованности при $t=0$ начального и граничных условий. Спустя непродолжительное время, можно ограничиться первым слагаемым: $\bar{c}(t) \approx 8 \exp \{-t / \tau\} / \pi^{2}\left(t>t_{0}>0\right)$, где параметр $\tau$ приобретает смысл времени релаксации (уменьшения в $e$ раз). Чтобы компенсировать отброшенные слагаемые и соответствовать начальным данным $\bar{c}(0)=c_{0}=1$, увеличим коэффициент с $8 / \pi^{2}$ до 1 . В итоге для аппроксимации $X(t) \approx \bar{c}(t)(t \geq 0)$ примем модель $\dot{X}(t)=-X(t) / \tau, X(0)=X_{0}=1$, и возвратимся к исходным переменным $(t, x): \dot{X}(t)=-K(T) X(t)$, $X_{0}=1, K(T)=\pi^{2} h^{-2} D(T)$. Здесь мы оставили нормировку $\quad \bar{c}(t)=\int_{0}^{h} c(t, x) d x / h \rightarrow \bar{c}(t) / c_{0}, \quad$ т. е. $\quad$ теперь $X(t) \in(0,1)$ - усредненная доля (от исходной равномерной концентрации водорода $\left.c_{0}\right)$, оставшаяся в образце к моменту $t>0$. Модель работоспособна, когда нас интересует интегральный поток десорбции без детализации физико-химических процессов. Интерпретация $K(T)$ позволяет для пористых и порошкообразных материалов говорить обобщенно об эффективных характеристиках переноса: коэффициенте диффузии $D$ и характерном пробеге $h$.

Можно не связывать $K(T)$ с диффузией, ограничившись выводом уравнения на уровне „скорость десорбции пропорциональна текущей усредненной концентрации“. Постулируется аррениусовская зависимость от температуры: $K(T)=K_{0} \exp \{-Q / R T\}$.

$\mathrm{C}$ другой стороны, в теории адсорбции-десорбции используется уравнение Поляни-Вигнера $\dot{\theta}=-M(T) \theta^{n}(t)$, где $\theta-$ степень заполнения поверхности. В частности, если речь идет о термодесорбции диссоциативно хемосорбированного двухатомного газа, то $n=2$. Модель можно адаптировать и для усредненной по объему концентрации, используя понятие эффективного коэффициента рекомбинации [3]. Для этого уравнение $d \bar{c} / d t=-b(T) \bar{c}^{2}$ разделим на $c_{0}$ и получим $\dot{X}=-K(T) X^{2}$, где $K=b c_{0}$. При этом с ростом давления начального насыщения образца водородом из газовой фазы температура максимума модельного потока десорбции будет уменьшаться.

В широком диапазоне условий эксперимента диффузия и десорбция с поверхности существенно взаимозависимы (динамика процессов на поверхности диктует граничные условия для уравнения диффузии). Такие более детализированные модели уже требуют разработки специализированного математического обеспечения $[19,20]$.

Синтезируя приведенные рассуждения, рассмотрим усредненную модель (с учетом линейного равномерного 
нагрева $\left.T(t)=T_{0}+\beta t, d T=\beta d t\right)$ :

$$
\begin{gathered}
\frac{d X}{d T}=-\beta^{-1} K(T) X^{\alpha}(T), X\left(T_{0}\right)=1, \\
K(T)=K_{0} \exp \left\{-Q[R T]^{-1}\right\}, \quad T \in\left[T_{0}, T_{*}\right] .
\end{gathered}
$$

Здесь $\alpha \in[1,2], T_{0}$ - начальная температура (обычно комнатная), когда десорбция водорода практически отсутствует; $T_{*}$ - конечная температура (когда десорбция уже пренебрежимо мала на фоне максимума потока); безразмерная переменная $X(T)$ имеет смысл доли усредненной по объему образца концентрации $\bar{c}$ от концентрации равномерного начального насыщения $c_{0}$. В силу $t \leftrightarrow T$ можно записать $X(T)=\bar{c}(T) / c_{0}$. Кинетический коэффициент $K(T) \quad([K]=1 / \mathrm{s})$ заранее не связываем явной формулой с коэффициентом диффузи $D(T)$ или коэффициентом рекомбинации $b(T)$. Если предполагается обрабатывать данные с различными условиями равномерного насыщения, то целесообразно явно выделить зависимость от $c_{0}$ в форме $K=K_{c}(T) c_{0}^{\alpha-1}$.

Дробный (континуальный) параметр $\alpha \in[1,2]$ позволяет учитывать степень участия лимитирующих факторов. Применяем усреднение не только по концентрации, но и по процессам диффузии и рекомбинации. Параметр $\alpha \in[0,1]$ можно было бы ввести в форме $\dot{X}=-\left[\alpha K_{1}(T) X+(1-\alpha) K_{2}(T) X^{2}\right]$, но параллельные каналы дегазации, лимитированные независимо диффузией и объемной рекомбинацией, представляются менее адекватной моделью. Усредненные по концентрации модели удовлетворительно описывают основную часть изолированного всплеска на ТДС спектре.

\section{2. Свойства модельного ТДС пика}

\section{1. Унимодальность и методика Киссинджера}

Нас интересует зависимость $w(T) \equiv-d X / d T=$ $=K(T) X^{\alpha}(T) / \beta-$ нормированный поток термодесорбции (в долях $X=\bar{c} / c_{0}$ на градус температуры). Знак тождества (三) используем по контексту в смысле равенства по определению. Если оперируем временем, то рассматриваем $v(t) \equiv-\dot{X}(t)=K X^{\alpha}(t)$. При этом $w(T)=v(T) / \beta$ с учетом $t \leftrightarrow T=T_{0}+\beta t$. График $w(T)$ представляет ТДС спектр. Поскольку используется (безразмерная) нормировка $X\left(T_{0}\right)=1$, то должно быть $S \equiv \int_{T_{0}}^{T_{*}} w(T) d T=1$. Строго говоря, нужно формально интегрировать на $(0, \infty)$, но мы рассматриваем отрезок $\left[T_{0}, T_{*}\right]$, вне которого поток пренебрежимо мал. Итак, модельный ТДС пик нормирован по площади. Регистрируемый поток нормируется интегралом (он равен $\left.c_{0}\right)$. Интегрируя уравнение $d X / d T=-K X^{\alpha} / \beta$ в квадратурах, получаем

$$
\begin{aligned}
& w(T)=-d X / d T \\
& =\left\{\begin{array}{l}
\frac{K(T)}{\beta} \exp \left\{-\int_{T_{0}}^{T} \frac{K(\tilde{T})}{\beta} d \tilde{T}\right\}, \alpha=1 \\
\frac{K(T)}{\beta}\left[1+(\alpha-1) \int_{T_{0}}^{T} \frac{K(\tilde{T})}{\beta} d \tilde{T}\right]^{\frac{\alpha}{1-\alpha}}, \alpha \in(1,2] .
\end{array}\right.
\end{aligned}
$$

Представления согласованы: предельный переход $\alpha \rightarrow+1$ во второй формуле дает первую. Начальная температура $T_{0}$ относительно низкая (обычно комнатная, когда $K\left(T_{0}\right) \ll 1$ и поток еще пренебрежимо мал). С ростом температуры аррениусовский коэффициент $K(T)$ монотонно выходит на горизонтальную асимптоту, а множитель с интегралом от $K(T)$ монотонно убывает. Это дает колоколообразный график $w(T)$.

Далее производную по температуре $T$ (в отличие от точки сверху для обозначения производной по времени $t$ ) будем обозначать штрихом. Приведем результаты вычислений для $\alpha \in[1,2]$ (с учетом $\left.K^{\prime}=K Q / R T^{2}\right)$ :

$$
\begin{aligned}
& w^{\prime}(T)=w(T) \varphi(T), \\
& w^{\prime \prime}(T)=w(T)\left[\varphi^{2}(T)+\varphi^{\prime}(T)\right], \\
& w^{\prime \prime \prime}(T)=w(T)\left[\varphi^{3}(T)+3 \varphi(T) \varphi^{\prime}(T)+\varphi^{\prime \prime}(T)\right] .
\end{aligned}
$$

Здесь приняты обозначения

$$
\begin{aligned}
& \varphi(T) \equiv Q R^{-1} T^{-2}-\alpha \beta^{-1} K(T) \hat{K}(T), \\
& \hat{K}(T ; \alpha) \equiv\left[1+(\alpha-1) \beta^{-1} \int_{T_{0}}^{T} K(\tilde{T}) d \tilde{T}\right] .
\end{aligned}
$$

В контексте обратной задачи параметрической идентификации, рассмотренной ниже, отметим, что $\hat{K}(T ; \alpha)=X^{1-\alpha}(T), \hat{K}(T ; 1)=1$. Для поиска температуры $T_{m}=T_{\max }$ экстремума (который является единственным, глобальным максимумом) запишем уравнение $w^{\prime}\left(T_{m}\right)=0$, откуда с учетом $w^{\prime}=0 \Leftrightarrow \varphi=0$ получаем $z \equiv Q\left[R T_{m}\right]^{-1}$,

$$
K\left(T_{m}\right)=\beta z\left[\alpha T_{m}\right]^{-1} \hat{K}\left(T_{m} ; \alpha\right) .
$$

Выделение безразмерной переменной $z$ в выкладках целесообразно, поскольку дробь вида $-E / R T$ фигурирует в экспоненте закона Аррениуса. В дальнейшем рассмотрении потребуются последующие производные:

$$
\begin{gathered}
w^{\prime \prime}\left(T_{m}\right)=w\left(T_{m}\right) \varphi^{\prime}\left(T_{m}\right)=\frac{w\left(T_{m}\right) z}{T_{m}^{2}}\left[-\frac{z}{\alpha}-2\right]<0, \\
w^{\prime \prime \prime}\left(T_{m}\right)=w\left(T_{m}\right) \varphi^{\prime \prime}\left(T_{m}\right)=\frac{w\left(T_{m}\right) z}{T_{m}^{3}}\left[\frac{\alpha-2}{\alpha^{2}} z^{2}+2 z+6\right] .
\end{gathered}
$$

Рассмотрим сначала (1) как уравнение для $T=T_{m}$. Перепишем его в форме

$$
f(T) \equiv \alpha T^{2} K(T)-(\alpha-1) \frac{Q}{R} \int_{T_{0}}^{T} K d \tilde{T}=\frac{\beta Q}{R} .
$$




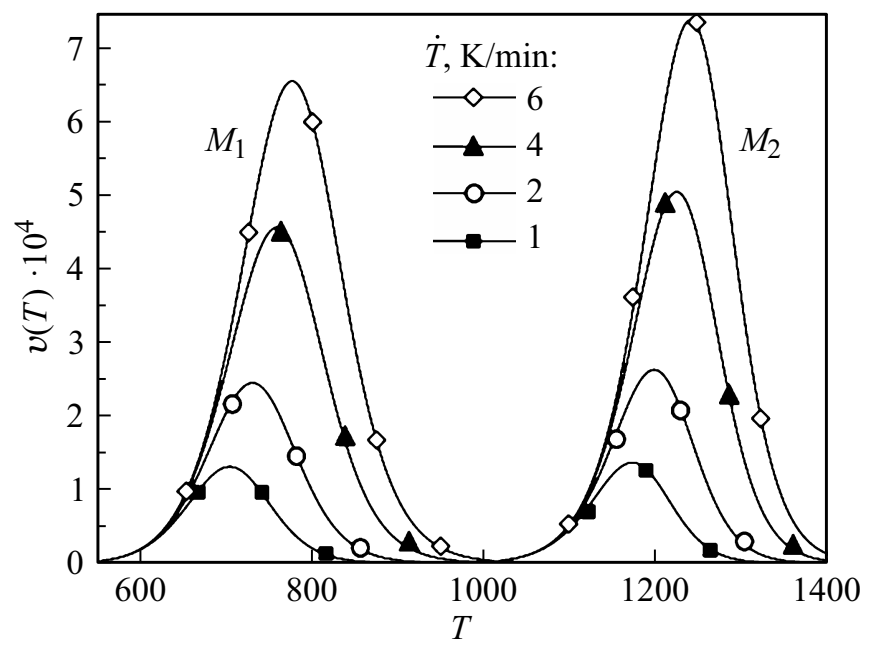

Рис. 1. Спектры $(T, v)$, вариация $\beta$.

Геометрически требуется найти пересечение графика $f(T)$ с горизонтальной прямой, определяемой значением $\beta Q / R$. Поскольку из соотношений $K(T)=K_{0} \exp \{-Q / R T\}, \quad K(T)=K(T) Q /\left[R T^{2}\right] \quad$ следует неравенство $f^{\prime}(T)=K(T)[2 \alpha T+Q / R]>0$ (положительна и вторая производная), решение единственно. Существование обеспечивается условием $K\left(T_{0}\right) \ll 1$ (формально можно рассматривать $T_{0}=0$ и $\left.T>0\right)$. Увеличение $\beta$ (правой части (4)) смещает температуру максимума $T_{m}$ вправо.

Уточним априорное ограничение на выбор предэкспоненты (частотного множителя) $K_{0}$, диктуемое уравнением (4). Значение $T_{0}$ определено как температура (обычно комнатная), при которой поток еще незначителен на фоне предстоящего максимума при $T=T_{m}>T_{0}$. Поэтому в рамках модели ТДС пика должно быть $f\left(T_{0}\right)<\beta Q / R$, т. е. $K_{0}<\beta Q \exp \left\{Q / R T_{0}\right\} /\left[\alpha R T_{0}^{2}\right]$. Подчеркнем, что зависимость $T_{m}\left(K_{0}, Q ; \alpha, \beta\right)$ определяется алгоритмически решением уравнения (4), т.е. у переменной $z=\frac{Q}{R T_{m}}$ числитель и знаменатель не являются независимыми при фиксированных $K_{0}$.

Для иллюстрации фиксируем $\alpha=1.5$, скорости нагрева $6,4,2,1[\mathrm{~K} / \mathrm{min}]$ и наборы значений $M_{1}: Q=100 \mathrm{~kJ} / \mathrm{mol}$, $K_{0}=10^{4} 1 / \mathrm{s} ; M_{2}: Q=300, K_{0}=10^{10}$. Модельные спектры в осях $\{T, v=-\dot{X}\}(t \leftrightarrow T)$ приведены на рис. 1 . Скорости нагрева указаны в порядке убывания максимумов. Набору $M_{2}$ соответствует правое семейство кривых. Наблюдается склейка восходящих фронтов, $T_{m}$ сдвигается вправо. Рис. 2 отражает зависимость спектра от модельного параметра $\alpha$ (на этот раз в осях $\{T, w=-d X / d T\})$. Восходящий фронт стабилен. Нисходящий фронт становится более пологим с ростом $\alpha$ (взяты значения $\beta=0.1[\mathrm{~K} / \mathrm{s}], \alpha=1,1.3,1.6,2$.

На практике (при $\alpha=1$ ) для оценки $Q, K_{0}$ используют зависимость значения $T_{m}$ от скорости нагрева $\beta$. Перепишем соотношение (1) (для общего случая $\alpha \in[1,2]$ ) с использованием координат Киссинджера $\left\{1 / T, \ln \left[\beta / T^{2}\right]\right\}$ :

$$
\begin{gathered}
\ln \frac{\beta}{T_{m}^{2}}=-\frac{Q}{R} \frac{1}{T_{m}}+\ln \frac{R K_{0}}{Q}+\varepsilon(\cdot), \\
\varepsilon\left(K_{0}, Q ; \alpha, \beta\right) \equiv \ln \left[\alpha \hat{K}^{-1}\left(T_{m} ; \alpha\right)\right],
\end{gathered}
$$

$\hat{K}^{-1}\left(T_{m} ; \alpha\right)=X^{\alpha-1}\left(T_{m}\right),\left.\varepsilon(\cdot)\right|_{\alpha=1}=0$. В подобных выражениях под знаком логарифма лишь числовые значения. При $\alpha=1$ имеем прямую $(\varepsilon=0)$, что позволяет с помощью линейной регрессии оценить коэффициент наклона прямой $(-Q / R)$ и затем параметр $K_{0}$. Существенное отклонение от прямой позволяет включить в процедуру оценивания и параметр $\alpha \in(1,2]$ (увеличение которого интерпретируем как рост влияния ассоциативной десорбции).

По экспериментальным данным $\left\{\beta_{j} ; T_{m j}\right\}$ вычисляем „откорректированные“ значения $\gamma_{j}=\ln \left[\beta_{j} / T_{m j}^{2}\right]-\varepsilon_{j}$ в левой части (5) и линейной регрессией оцениваем коэффициенты $a_{1}=-Q / R$ и $a_{2}=\ln \left[R K_{0} / Q\right]\left(\Rightarrow Q, K_{0}\right)$. При этом

$$
\begin{aligned}
& \hat{K}^{-1}\left(T_{m}, \alpha\right)=X^{\alpha-1}\left(T_{m}\right) \Rightarrow \\
& \varepsilon_{j}=\ln \alpha+(\alpha-1) \ln X\left(T_{m j}\right),
\end{aligned}
$$

а значение $X\left(T_{m}\right)$ вычисляется как интеграл от $w(T)$ по $T \in\left[T_{m}, T_{*}\right]$. Зависимость от параметра $\alpha$ „медленная“, так что для его подбора можно просто просканировать диапазон $[1,2]$ с шагом 0.1-0.2, „выпрямляя“ множество $\left\{\gamma_{j}\right\}$ в осях $\{1 / T, \gamma\}$. Заметим, что если пики близки к симметричным, то $X\left(T_{m}\right) \approx 1 / 2$, что приведет к линейному вырождению $\left(\varepsilon_{j}(\alpha)\right.$ не зависят от $\left.j\right)$. Оценку энергии активации $Q$ это не изменит, а для уточнения значения $K_{0}=K_{0}(\alpha)$ (по коэффициенту $a_{2}=a_{2}(\alpha)$ ) потребуется сопоставление экспериментального и модельного пиков в целом.

Итак, модификация метода Киссинджера состоит в следующем.

1.Пусть наблюдаются унимодальные пики, и данные $\left\{\beta_{j}, T_{m j}\right\}$ удовлетворительно аппроксимируются прямой

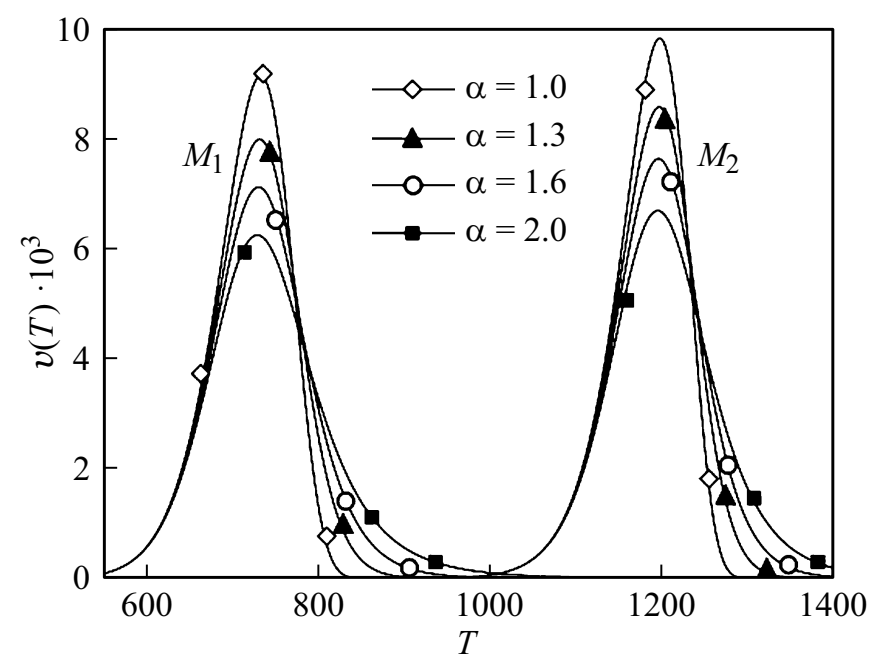

Рис. 2. Спектры $(T, w)$, вариация $\alpha$. 
в координатах $\left\{1 / T, \ln \left[\beta / T^{2}\right]\right\}$. Если принимаем $\alpha=1$ (в том числе и в качестве гипотезы), то применяем классическую схему.

2. Если пики практически симметричны $\left(X\left(T_{m}\right) \approx 1 / 2\right)$, но усредненный порядок реакции $\alpha \in[1,2]$ априори неизвестен, то можно однозначно оценить $Q$ и найти зависимость $K_{0}(\alpha)$ линейной регрессией в координатах $\{1 / T, \gamma\}$. При этом $\varepsilon_{j} \equiv \varepsilon=\ln \alpha-(\alpha-1) \ln 2$.

3. Если отклонение от прямой (п. 1) существенно, но выбором $\alpha$ можно получить $\{1 / T, \gamma\}-$ прямую, то $\alpha$, $Q, K_{0}$ оцениваются однозначно.

В любом случае затем нужно сравнить экспериментальные и модельные спектры (численное решение ОДУ) в целом, поскольку формула Киссинджера выведена в предположении, что десорбция описывается „монореакцией“ для усредненной концентрации. Данные о сдвиге температуры $T_{m}$ при разных скоростях нагрева - лишь часть информации о ТДС спектре. В случае многопикового спектра проводится разложение на сумму пиков. Эта более сложная задача требует отдельного рассмотрения.

\section{2. Анализ симметрии ТДС пика}

Одной из общепринятых методик является разложение спектра на сумму гауссовских кривых (например, в пакете прикладных программ Origin). Более адекватным является разложение на сумму ТДС пиков, генерируемых реакциями 1-2 порядков. Но они, строго говоря, несимметричны. Возникает задача поиска критериев симметричности и диапазонов параметров, где отклонения от гауссианов практически несущественны.

Качественное отличие ТДС пика $(\alpha \in[1,2])$ от гауссовской кривой состоит лишь в возможной существенной несимметричности относительно вертикали $T=T_{m}$. В этом случае спектр хорошо аппроксимируется склейкой двух гауссианов:

$$
G_{1,2}(T)=w\left(T_{m}\right) \exp \left\{-\frac{\left(T-T_{m}\right)^{2}}{2 \sigma_{1,2}^{2}}\right\}, T \gtrless T_{m} .
$$

Следуя „правилу двух сигм“, такую аппроксимацию достаточно рассматривать на отрезке $\left[T_{m}^{-}, T_{m}^{+}\right] \equiv\left[T_{m}-2 \sigma_{1}, T_{m}+2 \sigma_{2}\right] \cap\left[T_{0}, T_{*}\right]$. Практический интерес представляет центральная часть спектра; начальный и конечный этапы (когда поток незначителен на фоне максимума) малоинформативны. Значения $\sigma_{1,2}$ находятся из соотношений

$$
\begin{aligned}
G_{1,2}\left(T_{1,2}\right) & =w\left(T_{m}\right) \exp \{-2\}, T_{1,2} \gtrless T_{m} \\
& \Rightarrow 2 \sigma_{1}=T_{m}-T_{1}, \quad 2 \sigma_{2}=T_{2}-T_{m} .
\end{aligned}
$$

Численные эксперименты в широком физически обоснованном диапазоне значений $\left\{K_{0}, Q\right\}$ [2] показывают, что в той или иной мере модельный ТДС спектр несимметричен. Это соответствует экспериментальным данным. Для пика, определяемого реакцией первого порядка $(\alpha=1)$, возможен вариант „относительно крутой восходящий фронт и пологий нисходящий“', но обычно пологий восходящий фронт сменяется крутым нисходящим. Для спектра, соответствующего реакции второго порядка $(\alpha=2)$, характерен относительно крутой восходящий фронт и более пологий нисходящий. Существенная асимметрия встречается как при $\alpha=1$, так и при $\alpha=2$.

Перейдем к рассмотрению критерия симметричности графика $w(T)$ относительно вертикали $T=T_{m}$. Для этого воспользуемся разложением

$$
\begin{aligned}
w\left(T_{m}+\Delta\right) & =w\left(T_{m}\right)+w^{\prime}\left(T_{m}\right) \Delta+\frac{1}{2} w^{\prime \prime}\left(T_{m}\right) \Delta^{2} \\
& +\frac{1}{6} w^{\prime \prime \prime}\left(T_{m}\right) \Delta^{3}+\frac{1}{24} w^{(4)}\left(T_{m}\right) \Delta^{4}+o\left(\Delta^{4}\right) .
\end{aligned}
$$

Численные эксперименты подтверждают, что в физически реальном диапазоне $T \in\left[T_{m}^{-}, T_{m}^{+}\right]$пятую производную уже можно не учитывать. В экстремуме $w^{\prime}\left(T_{m}\right)=0$, а слагаемые с $\Delta^{2}$ и $\Delta^{4}$ дадут те же (симметричные) значения при замене $\Delta$ на $-\Delta$. Для оценки симметричности ТДС пика достаточно оценить производную $w^{\prime \prime \prime}\left(T_{m}\right)$ в сравнении с $w^{\prime \prime}\left(T_{m}\right)$. Критерий симметричности $\left|w^{\prime \prime}\left(T_{m}\right)\right| \gg \frac{\left|w^{\prime \prime \prime}\left(T_{m}\right)\right||\Delta|}{3}, \Delta \in\left[-2 \sigma_{1}, 2 \sigma_{2}\right]$, где $T_{m}=T_{m}\left(K_{0}, Q ; \alpha, \beta\right), \sigma_{i}=\sigma_{i}\left(T_{m}, Q ; \alpha, \beta\right)$, преобразуется к виду

$$
\frac{|\Delta|}{3 T_{m}} \cdot \frac{\left|6+2 z-\alpha^{-2}(2-\alpha) z^{2}\right|}{2+z} \ll 1, z \equiv \frac{Q}{R T_{m}} .
$$

Численный анализ показывает, что из рассматриваемых допустимых значений $\alpha$ и $z$ (в соответствии с табл. 1 в [2]) только относительно малая часть оказывается в области, где соотношение параметров дает практически симметричный спектр. Отметим, что при $\alpha=2$ заведомо $w^{\prime \prime \prime}\left(T_{m}\right)=w\left(T_{m}\right)(2 z+6) z / T_{m}^{3}>0$, что объясняет выводы численных экспериментов о несимметричности ТДС спектра реакции второго порядка (восходящий фронт ниже). Вопрос лишь в том, насколько это отклонение существенно в конкретной задаче. Для $\alpha<2$ спектры, близкие к симметричным, получаются при $w^{\prime \prime \prime}\left(T_{m}\right) \approx 0$, откуда $z(\alpha) \approx \alpha^{2}\left[1+\sqrt{1+6 \alpha^{-2}(2-\alpha)}\right] /(2-\alpha)$. Формально $z(\alpha) \rightarrow+\infty$ при $\alpha \rightarrow 2-$, но гипотетическое превышение порога $z>20-30$ требует обоснования.

В частности, для реакции первого порядка $z=Q /\left[R T_{m}\right] \approx 1+\sqrt{7} \quad(\alpha=1), \quad$ что $\quad$ соответствует относительно низким значениям энергии активации $Q$. Даже если выполняется равенство $w^{\prime \prime \prime}\left(T_{m}\right)=0$ $\left(z^{2}-2 z-6=0\right), \quad$ то при соответствующем значении $z$ выполняется $w^{(5)}\left(T_{m}\right)=w\left(T_{m}\right) z T_{m}^{-5} p^{4}(z)$, $p_{4}(z) \equiv\left(z^{2}-2 z-6\right)\left(9 z^{2}+20 z-42\right)-60 z-132<0$.

Строго говоря, спектр всегда несимметричен. Поскольку третья производная $w^{\prime \prime \prime}\left(T_{m}\right)$ может менять знак (в широком диапазоне параметров), более пологой может 


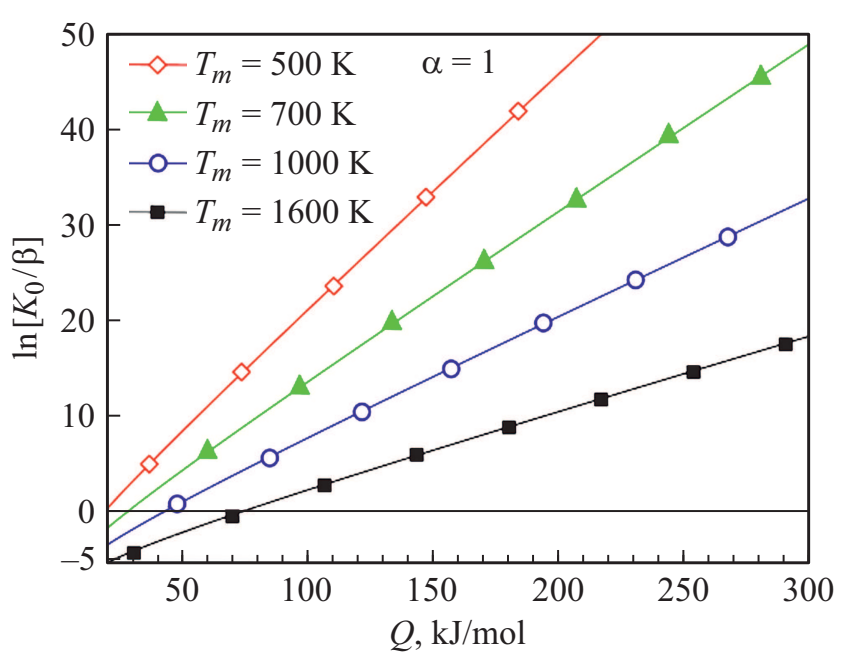

Рис. 3. Зависимость $\ln \left[K_{0} / \beta\right]$ от $Q, T_{m}$.

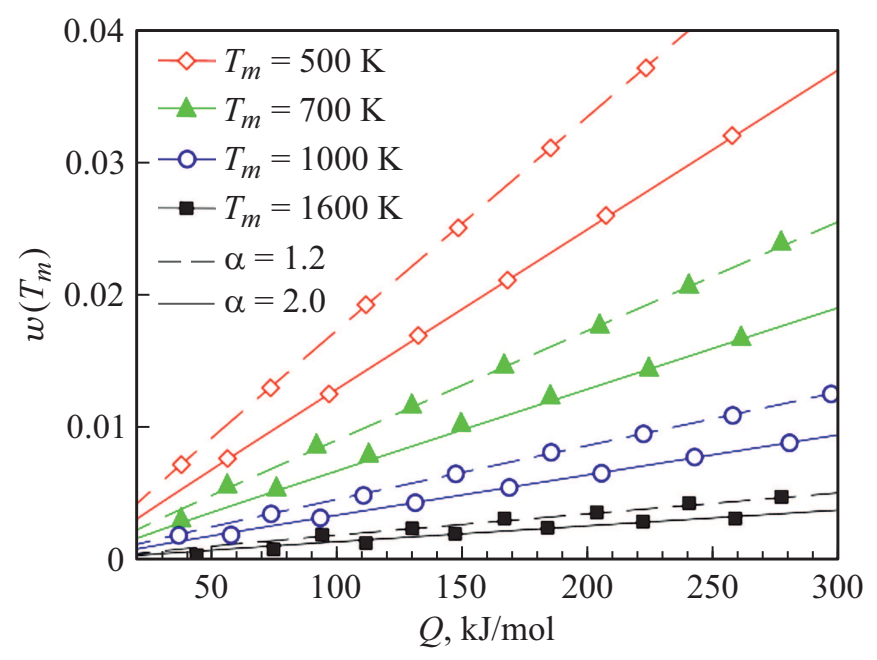

Рис. 4. Зависимость $w\left(T_{m}\right)$ от $Q, T_{m}, \alpha$.

оказаться как восходящая, так и нисходящая ветви модельного спектра.

Входными параметрами модели являются $\alpha, \beta, K_{0}$, $Q$. В эксперименте, когда ТДС пик явно выражен, значение $T_{m}$ известно. В силу уравнения (4) для определения температуры максимума $T=T_{m}$, которое имеет вид $K_{0} \cdot F\left(T_{m}, Q ; \alpha\right)=\beta Q / R$, можно явно выразить $K_{0}=K_{0}\left(T_{m}, Q ; \alpha, \beta\right)=\beta Q / R F$. В качестве варьируемых параметров остаются $\alpha$ и $Q$. В частности, при $a=1$ имеем выражение $K_{0}=\beta z \exp \{z\} / T_{m}$. Отсюда следует, что при неизменном $K_{0}$ росту $T_{m}$ соответствует увеличение энергии $Q$. Модельный пик становится все более узким. В контексте обратной задачи идентификации модели по измерениям входными данными лучше считать набор $\left\{T_{m}, Q ; \alpha, \beta\right\}$.

Целесообразно выбрать следующую схему представления графического материала. Ориентируясь на используемые в настоящей работе экспериментальные данные, фиксируем характерные дискретные значения $T_{m}$. Энер- гия активации является самым „активным“ параметром, ему уделяем особое внимание. Предэкспонента $K_{0}$ считается заданной формулой $K_{0}=\beta Q / R F$ из линейного по $K_{0}$ уравнения (4). Нас интересует отображение $Q \rightarrow w\left(T_{m}\right)$ при различных $T_{m}, \alpha$. Формально лучше за основные независимые переменные вместо $K_{0}, Q$ взять $T_{m}, z\left(z=Q /\left[R T_{m}\right]\right)$, но явное указание энергии $Q$ (и экспериментальной температуры $T_{m}$ ) привычнее в специальной литературе, так что остановимся на паре $T_{m}, Q$. При этом $K_{0}=K_{0}\left(T_{m}, Q\right)$ в силу уравнения (4).

При фиксированном $Q$ спектр второго порядка шире, значения $w^{\prime \prime}\left(T_{m}\right)$ и $w^{\prime \prime \prime}\left(T_{m}\right)$ больше при $\alpha=2$, чем при $\alpha=1$. На рис. 3 показана зависимость $K_{0} / \beta$ от $Q$ и $T_{m}$ в логарифмической шкале. Характерная зависимость максимума потока от энергии активации и температуры пиков показана на рис. 4.

\section{3. Параметрическая идентификация}

Рассмотрим изолированный (унимодальный) ТДС пик. Нормированные по площади зависимости $v(t)$ и $w(T)$ считаем известными. Нормировка означает переход к единичным начальным данным для $X(t)=\bar{c}(t) / c_{0}$. Данные зашумлены, так что при обработке измерений будем использовать корректные вычислительные методы (интегрирование и метод наименьших квадратов). Фиксируем $\alpha \in[1,2]$. Этот параметр будем варьировать в зависимости от текущей оценки $K_{0}, Q$. В силу принятой модели

$$
\begin{aligned}
& v(t)=-\dot{X}(t)=K(T(t)) X^{\alpha}(t), X_{0}=X(0)=1, \\
& w(T)=-X^{\prime}(T)=\beta^{-1} K(T) X^{\alpha}(T), X\left(T_{0}\right)=1
\end{aligned}
$$

можно вычислить следующие величины $\left(t_{i} \leftrightarrow T_{i} \in\left(T_{0}, T_{*}\right)\right):$

$$
X\left(t_{i}\right)=\int_{t_{i}}^{t_{*}} v(t) d t=\int_{T_{i}}^{T_{*}} w(T) d T=X\left(T_{i}\right) .
$$

Конечные времена $t_{*}$ и $T_{*}$ - температура достаточно велики, чтобы считать $X\left(t_{*}\right)=X\left(T_{*}\right)=0$. Вместо набора $t_{i} \leftrightarrow T_{i}$ возьмем одно значение $T_{m}$, поскольку температура максимума известна из эксперимента и является ключевым параметром. Значения $w\left(T_{m}\right)$ и $X\left(T_{m}\right)$ позволяют вычислить $K\left(T_{m}\right)$ :

$$
\begin{aligned}
& w\left(T_{m}\right)=\beta^{-1} K\left(T_{m}\right) X^{\alpha}\left(T_{m}\right) \\
& \Rightarrow K\left(T_{m}\right)=\beta w\left(T_{m}\right) X^{-\alpha}\left(T_{m}\right) .
\end{aligned}
$$

С другой стороны, согласно условию экстремума (1), выполняется

$$
K\left(T_{m}\right)=\frac{\beta Q}{\alpha R T_{m}^{2}} \hat{K}\left(T_{m} ; \alpha\right)=\frac{\beta Q}{\alpha R T_{m}^{2}} X^{1-\alpha}\left(T_{m}\right) .
$$

Из этого уравнения выражаем энергию активации $Q$ и (в силу аррениусовости $K(T)$ ) получаем следующее 
решение обратной задачи:

$$
\begin{gathered}
Q=Q^{*}=\beta^{-1} \alpha R T_{m}^{2} K\left(T_{m}\right) X^{\alpha-1}\left(T_{m}\right), \\
K_{0}=K_{0}^{*}=K\left(T_{m}\right) \exp \left\{Q^{*}\left[R T_{m}\right]^{-1}\right\} .
\end{gathered}
$$

Заметим, что если унимодальный спектр практически симметричен относительно вертикали $T=T_{m}$, то можно без интегрирования $w(T)\left(T \in\left[T_{m}, T_{*}\right]\right)$ принять $X\left(T_{m}\right)=1 / 2$ (и тогда достаточно лишь значения $\left.w\left(T_{m}\right)\right)$. Для граничных $\alpha=1,2$ формально выражения совпадают. Для сопоставления с модельным спектром экспериментальный график нужно нормировать по площади (поделить на интеграл по $\left.T \in\left[T_{0}, T_{*}\right]\right)$. Температуры $T_{0}, T_{*}$ фиксируются условием $w\left(T_{0}\right)=w\left(T_{*}\right)=0$ (на фоне $\left.w\left(T_{m}\right)\right)$.

Если экспериментальные данные сильно зашумлены, то целесообразно применить усредняющую процедуру. Вычисляем несколько значений

$$
\begin{aligned}
& K_{i}=K\left(T_{i}\right)=\beta w\left(T_{i}\right) X^{-\alpha}\left(T_{i}\right), \\
& X\left(T_{i}\right)=\int_{T_{i}}^{T_{*}} w(T) d T=1-\int_{T_{0}}^{T_{i}} w(T) d T,
\end{aligned}
$$

и решаем задачу линейной регрессии (выбором $\ln K_{0}$ и $Q$ ) из соотношений

$$
\ln K_{i}=\ln K_{0}-\left[R T_{i}\right]^{-1} Q, 1 \leq i \leq n .
$$

Если предметом идентификации считать не только параметры, но и саму модель, хорошее совпадение с прямой в координатах $\left\{\ln K, 10^{3} / T\right\}$ является лишь косвенным подтверждением адекватности принятой модели (см. разд. 4).

Значение параметра $\alpha$ настраиваем из следующих соображений. После остановки нагрева при $\alpha=1$ модельный поток падает экспоненциально. Следовательно, в логарифмической шкале должна быть прямая. Наличие прогиба дает основание увеличивать этот параметр в диапазоне $(1,2]$ (с шагом 0.1-0.2). Выбор $\alpha$ влияет на значения (9) и решение системы (10). В итоге останавливаемся на варианте с наименьшим среднеквадратическим отклонением. Решение зависит и от набора $t_{i} \leftrightarrow T_{i}$. Представляется целесообразным при каждом $\alpha$ (и соответствующих значениях $K_{0}, Q$ ) численно интегрировать уравнение $X^{\prime}=-\beta^{-1} K X^{\alpha}\left(X\left(T_{0}\right)=0\right)$ и сравнивать модельный спектр с экспериментальным на всем отрезке $\left[T_{0}, T_{*}\right]$.

Отметим особенность, когда поток регистрируется лишь в относительных единицах. Предварительно нормируем спектр по площади, поделив на интеграл. При $\alpha>1$ возникает неопределенность в коэффициенте $K=K\left(T ; c_{0}\right)=K_{c}(T) c_{0}^{\alpha-1}$, поскольку теперь начальная концентрация известна лишь с точностью до множителя. Этот множитель формально войдет в $K_{0}$. А энергия активации $Q$ определяется однозначно.
Если экспериментальный ТДС спектр составной, состоящий из суммы нескольких локальных пиков первого порядка (такое предположение обычно постулируется), то задача идентификации усложняется. Для начального приближения можно использовать стандартные симметричные гауссианы. Это дает, в частности, информацию о примерных значениях $T_{m}$ и начальных долях каждого моделируемого всплеска (а это дополнительные параметры варьирования в общей начальной доле $X_{0}=1$ ). Имеются в виду не только ярко выраженные экспериментальные локальные ТДС пики, но и элементы разложения составных (относительно широких) локальных пиков на сумму гауссианов. При моделировании ТДС пиков первого порядка используется соотношение $K\left(T_{m}\right)=K_{0} \exp \left\{-Q / R T_{m}\right\}=\beta Q / R T_{m}^{2}$, что позволяет при задаваемых $T_{m}$ варьировать лишь переменную $Q$ и соответствующую часть начальной суммарной единичной доли в физически обоснованных диапазонах.

\section{4. Модель с динамическими граничными условиями}

Выше рассмотрена модель динамики десорбции в терминах усредненной по объему образца концентрации. Перейдем к более детализированной модели, явно разделяя объемные и поверхностные процессы (следуя работе [1], стр. 177-206). Вакуумную систему считаем достаточно мощной, чтобы пренебречь ресорбцией. Для тонкой однородной пластины толщины $l$ в условиях медленного равномерного нагрева краевая задача ТДС дегазации примет следующий вид:

$$
\begin{gathered}
c_{t}(t, x)=D(T) c_{x x}(t, x), t \in(0, t), x \in(0, l), \\
c(0, x)=c_{0}, x \in[0, l], c_{0, l}(t)=g(T) q(t), \\
\dot{q}(t)=-b(T) q^{2}(t)+D(T) c_{x}(t, 0), \\
J(T)=b(T) q^{2}(t), T(t)=T_{0}+\beta t, \beta>0 .
\end{gathered}
$$

Здесь $c(t, x)$ - концентрация растворенного водорода; $q(t)$ - поверхностная концентрация; $D, b, g-$ (аррениусовские) коэффициенты диффузии, десорбции, быстрого растворения (локального квазиравновесия поверхностной и приповерхностной объемной концентрации); $J(T)$ - плотность потока десорбции (атомов, рекомбинировавших в молекулы). В силу $t \leftrightarrow T$ используем также упрощенную запись $J(t) \equiv J(T(t))$.

Алгоритм решения краевых задач термодесорбции на основе разностных схем (включая учет обратимого захвата диффузанта различного рода ловушками) подробно описан в [19]. Аппроксимация в классе ODE представлена в [20]. Раздвоение (бифуркация) спектра появляется при определенном соотношении энергий активации диффузии и десорбции, когда ни один из процессов не является строго лимитирующим и проявляется взаимообусловленность их динамики. В модели для усредненной концентрации параметр $\alpha \in[1,2]$ 


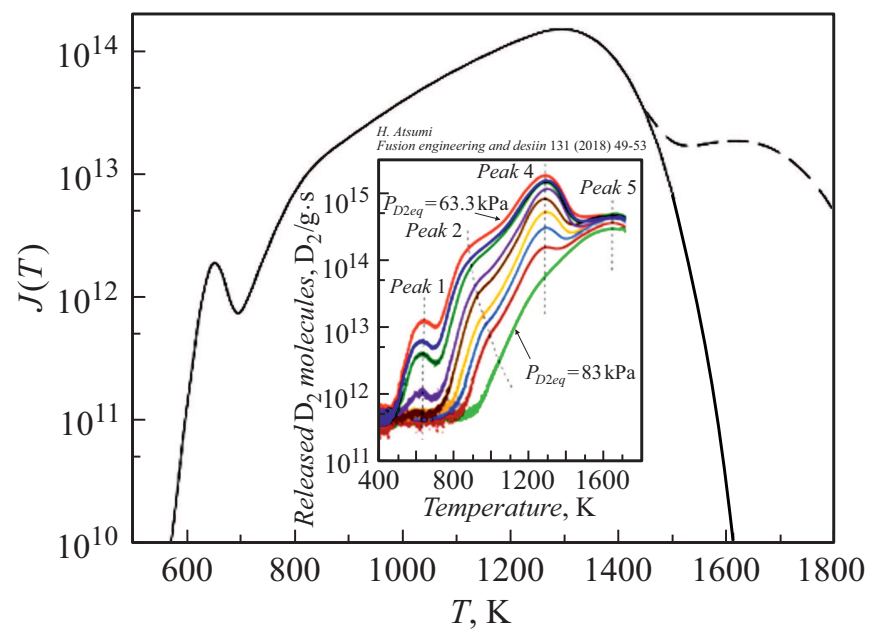

Рис. 5. Моделирование спектра [25].

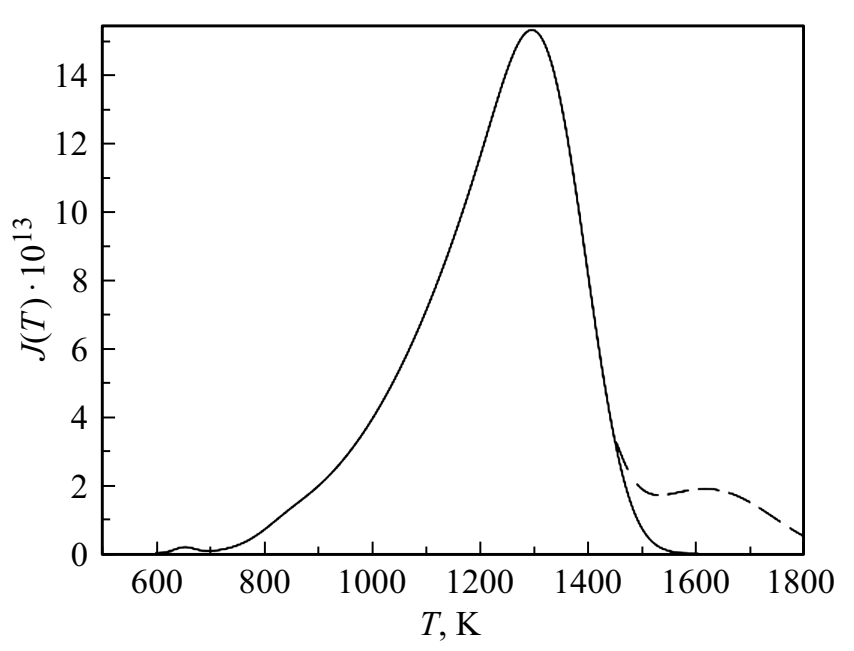

Рис. 6. Спектр [25] в линейной шкале.

позволяет учитывать лишь интегрально вклад (доли) диффузионных и поверхностных процессов.

Модель с динамическими условиями на поверхности позволяет воспроизвести характерный вид всего диапазона „Peak 1-Peak 4“ составного экспериментального спектра в работе [25] (см. рис. 5 [25]). Необходима оговорка: в [25] речь о графите, а приведенная здесь модель скорее характерна для металлических пластин. Но на уровне выявления общих качественных закономерностей можно придавать коэффициентам десорбции и диффузии в графите некоторый эффективный смысл.

Результаты моделирования представлены на рис. 5 в логарифмической шкале, как в оригинальной работе. Дополнительно спектр изображен на рис. 6 в линейной шкале. Фиксированные параметры: $l=0.1 \mathrm{~cm}$, $b_{0}=10^{3} \mathrm{~cm}^{2} / \mathrm{s}, E_{b}=250 \mathrm{~kJ} / \mathrm{mol}, D_{0}=0.1 \mathrm{~cm}^{2} / \mathrm{s}, E_{D}=125$, $g_{0}=10^{4} \mathrm{~cm}^{-1}, \quad E_{g}=0, \quad c_{0}=4.8 \cdot 10^{18} \mathrm{~cm}^{-3}, \quad T_{0}=300 \mathrm{~K}$, $\dot{T}=\beta=0.1 \mathrm{~K} / \mathrm{s}$. Переход к линейным координатам на рис. 6 показывает несопоставимость масштабов пиков, обозначенных номерами 2 и 3 на рис. 1,2,5,6 [25], с основным ТДС всплеском. Выраженными эти „пики“ становятся лишь при деформации спектра при переходе к логарифмической шкале.

Природа модельного пика 2-3 (800-1200 К) известна. После десорбции с поверхности и из подповерхностного слоя поток локально падает (пик 1). Затем поток начинает снова расти. Причиной этого является дальнейший нагрев и образовавшийся большой градиент концентрации у поверхности, что активизирует диффузию. Падение градиента замедляет скорость диффузионной подкачки и образуется естественное плечо 2-3, которое не является следствием каких-либо реакций 1-2 порядков в объеме (ловушек с соответствующими энергиями связи нет). Это в модели, когда ответ на вопрос о причинах изгибов спектра известен после решения краевой задачи. Модель способна описать сразу диапазон „Пиков“ (рис. 5). Приписывание каждому экспериментальному локальному всплеску энергий связи в объеме требует дополнительного обоснования.

При описании множества ТДС пиков удобна модель

$$
\begin{gathered}
\partial_{t} c=D \partial_{x}^{2} c-\sum_{v=1}^{m}\left[a_{v}^{-1}\left[1-Z_{v}\right] c(t, x)-a_{v}^{+} z_{v}(t, x)\right], \\
\partial_{t} z_{v}=a_{v}^{-}(T)\left[1-Z_{v}\right] c(t, x)-a_{v}^{+}(T) z_{v}(t, x),
\end{gathered}
$$

где $z_{v}(t, x)$ - концентрации атомов водорода, захваченного дефектами различных типов; $a_{v}^{\mp}-$ коэффициенты поглощения и выделения $H$ ловушками; $Z_{v} \equiv z_{v}(t, x) / z_{\max }^{v}-$ степень насыщения $\left(z_{\max }^{v}=\max z_{v}\right)$. Для практических целей захват учтен в простейшей „интегральной“ форме, уточнение геометрии дефектов и их распределения существенно усложнило бы модель. Если дефект, например, не микрополости, а включения гидридной фазы, то на этапе дегазации соответствующий коэффициент $a_{j}^{-}(T)$ тождественно равен нулю, а значение $a_{j}^{+}(T)$ положительно лишь после достижения критической температуры: $T(t) \geq T_{\text {crit }}$. За счет различных энергий связи (коэффициентов $E_{a}$ ) можно добиться заданного количества пиков.

Например, для модельного воспроизведения пологого высокотемпературного пика 5 при $t>1400$ (см. рис. 5 [25] или врезку на рис. 5 настоящей работы) в краевую задачу достаточно добавить одну ловушку типа „разложения фазы“ (в процессе дегазации $a^{-}=0$ ), динамика концентрации в которой определяется уравнением $\partial_{t} z(t, x)=-a^{+}(T) z(t, x)$ (реакция 1-го порядка). Коррекция спектра показана на рис. 5,6 штриховой линией. Параметры: $z(0)=10^{18} \mathrm{~cm}^{-3} ; a_{0}^{+}=50 \mathrm{~s}^{-1}$; $E_{a}=150 ; T_{\text {crit }}=1200 \mathrm{~K}$.

На рис. 7 представлено изменение спектра при вариации скорости нагрева $(6,4,2,1,0.5[\mathrm{~K} / \mathrm{min}]$ по убыванию максимума) для качественного сопоставления с экспериментальными данными, представленными на рис. 3 [26]. Плечо, обозначенное как „Peak 2“, заметно лишь в логарифмической шкале. Для модельного воспроизведения 


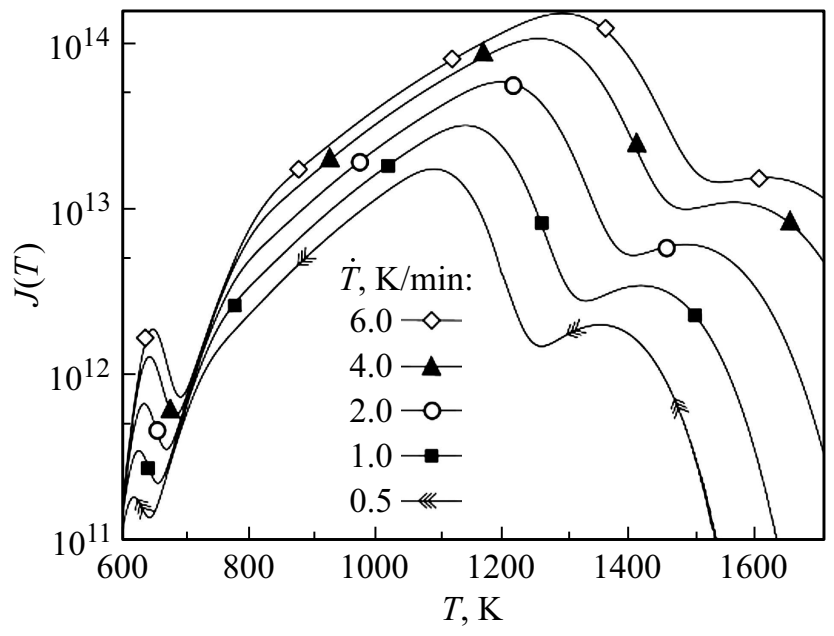

Рис. 7. Вариация скорости нагрева.

высокотемпературного пологого пика в краевую задачу достаточно добавить „ловушку“, начинающую выделять водород при достижении $T_{c r i t}=1200 \mathrm{~K}$. Но можно поступить и по-другому, если, например, имеется дополнительная экспериментальная информация о наличии тугоплавких оксидов на поверхности пластины. Они препятствуют десорбции водорода, но после достижения критической температуры поверхность от них освобождается. Эффективно это можно моделировать ростом площади поверхности или увеличением коэффициента десорбции $b$. Формально можно по достижении критической температуры начинать линейно уменьшать энергию активации: $E_{b}=E_{b}^{0}-a\left[T-T_{\text {crit }}\right], a>0$. Эффект на графике будет тем же - появится дополнительный высокотемпературный пик.

Воспримем теперь ТДС спектр, генерируемый динамической моделью, как экспериментальный, и применим к пикам $1,4,5$ (в нумерации [25], см. рис. 5,7) методику разложения на составляющие элементарные реакции и методику Киссинджера. Соответствующие этим локальным пикам температуры: $T_{1-3} \approx 650,1300$, 1600 К. Плечо 2-3, заметное лишь в логарифмической шкале, относим к естественному замедлению диффузиидесорбции и не считаем его проявлением реакций в объеме.

Обработка „экспериментальных данных“ в априорном предположении наложения реакций 1-го порядка дает следующие результаты. Энергия активации, соответствующая $T_{1}$, имеет значение $264 \mathrm{~kJ} / \mathrm{mol}$. Это соответствует $E_{b}=250$, так что первый локальный пик спектра можно считать именно десорбционным. Второй выраженный пик $\left(T_{2}\right)$ дает диффузионное значение $125.3 \approx 125=E_{D}$. Наконец, высокотемпературному пику соответствует (естественно в силу модели) $150=E_{a}$. При этом в координатах Киссинджера $\left\{1 / T_{m}, \ln \left[\beta / T_{m}^{2}\right]\right\}$ имеем практически идеальные прямые.

Таким образом, в данном случае методика разложения на элементарные реакции (для усредненной концентра- ции) дает хорошие приближения, но не для энергий связи захваченного в объеме водорода, а для энергий активации физико-химических процессов десорбции, диффузии, разложения „гидридной фазы“ (или оксида на поверхности при соответствующем увеличении $b$ ) в их динамическом взаимодействии. В модели нет захвата, водород находится в диффузионно подвижном состоянии.

\section{Заключение}

В работе анализируются различные модели пиков термодесорбции водорода. Модель динамики усредненной по объему концентрации с континуальным параметром позволяет учесть интегрально степень доминирования лимитирующих факторов диффузии и рекомбинации атомов $H$ в десорбирующиеся молекулы. В частности, это позволяет оценить динамику изменения различных характеристик процесса дегазации при переходе от реакций первого порядка к реакциям второго порядка.

Предложен аналитический критерий оценки степени симметричности модельных ТДС пиков (унимодальных ТДС спектров реакций 1-2 порядков). Это важно для применимости методики разложения составного пика на сумму гауссианов.

Предложены модификации метода Киссинджера (для нескольких скоростей нагрева материала) и процедуры решения задачи параметрической идентификации пика лишь по одной скорости нагрева.

Сравнительный анализ с диффузионной моделью с динамическими граничными условиями, отражающими поверхностные процессы, показывает следующее. Нет априорной необходимости интерпретировать всплески спектра как следствие наличия ловушек в объеме с различными энергиями связи и описывать локальные пики реакциями 1-2 порядков для усредненной концентрации. Причиной локальных пиков может быть динамика взаимодействия объемных и поверхностных процессов, наличие окислов на поверхности... Нужны дополнительные экспериментальные данные и физические обоснования. Целесообразно провести дегазацию на пластинах разной толщины. Если пики остаются соизмеримыми по площади, то это аргумент в пользу моделей, оперирующими объемными характеристиками. Если первый пик практически не изменился, а последующий значительно возрос с увеличением толщины, то целесообразно взять за основу диффузионную модель в объеме с учетом поверхностных процессов (динамические граничные условия). При обоснованной необходимости в уравнение диффузии можно добавить слагаемые обратимого захвата, что лишь технически усложнит численное моделирование.

\section{Финансирование работы}

Исследование выполнено в рамках проекта РФФИ 1829-19149 мк. 


\section{Конфликт интересов}

Авторы заявляют, что у них нет конфликта интересов.

\section{Список литературы}

[1] Взаимодействие водорода с металлами, под ред. А. П. Захарова. (Наука, М., 1987)

[2] Ю.С. Нечаев. УФН, 176 (6), 581 (2006).

[3] А.А. Писарев, И.В. Цветков, Е.Д. Маренков, С.С. Ярко. Проницаемость водорода через металлы (МИФИ, М., 2008)

[4] Изотопь водорода. Фундаментальные и прикладные исследования, под ред. А.А. Юхимчука. (РФЯЦВНИИЭФ, Саров, 2009)

[5] Основы водородной энергетики, под ред. В.А. Мошникова, Е.И. Терукова. (ЛЭТИ, СПб., 2010)

[6] S. Vyazovkin, A.K. Burnham, J.M. Criado, L.A. PerezMaqueda, C. Popescu, N. Sbirrazzuoli. Thermochim. Acta, 520, 1 (2011). DOI: 10.1016/j.tca.2011.03.034

[7] S. Vyazovkin, K. Chrissafis, M.L. Di Lorenzo, N. Koga, M. Pijolat, B. Roduit, N. Sbirrazzuoli, J.J. Sunol. Thermochim. Acta, 590, 1 (2014). DOI: 10.1016/j.tca.2014.05.036

[8] M.V. Lototskyy, R. Denys, N.N. Serge, I. Bessarabskaia, V.A. Yartys. Mater. Today: Proc., 5 (4, 2), 10440 (2018). DOI: $10.1016 /$ j.matpr.2017.12.375

[9] F.G. Wei, M. Enomoto, K. Tsuzaki. Comput. Mater. Sci., 51, 322 (2012). DOI: 10.1016/j.commatsci.2011.07.009

[10] R. Silverstein, D. Eliezer, E. Tal-Gutelmacher. J. Alloys Compd., 747, 511 (2018). DOI: 10.1016/j.jallcom.2018.03.066

[11] Е.А. Денисов, Т.Н. Компаниец, А.А. Юхимчук, И.Е. Бойцов, И.Л. Малков. ЖТФ, 83 (6), 3 (2013). [E.A. Denisov, T.N. Kompaniets, A.A. Yukhimchuk, I.E. Boitsov, I.L. Malkov. Tech. Phys., 58 (6), 779 (2013). DOI: $10.1134 / \mathrm{S} 1063784213060091]$

[12] E.A. Hodille, L.B. Begrambekov, J.Y. Pascal, O. Saidi, J.M. Layet, B. Pegourie, C. Grisolia. Int. J. Hydrogen Energy, 39, 20054 (2014). DOI: 10.1016/j.ijhydene.2014.09.027

[13] L.B. Begrambekov, A. Evsin, A. Grunin, A. Gumarov, A.S. Kaplevsky, N. Kashapov, A. Luchkin, I. Vakhitov, I. Yanilkin, L.R. Tagirov. Int. J. Hydrogen Energy, 44, 17154 (2019). DOI: 10.1016/j.ijhydene.2019.04.198

[14] F.J. Castro, G. Meyer. J. Alloys Compd., 330-332, 59 (2002). DOI: $10.1016 / \mathrm{S} 0925-8388(01) 01625-5$

[15] E.A. Evard, I.E. Gabis, V.A. Yartys. Int. J. Hydrogen Energy, 35, 9060 (2010). DOI: 10.1016/j.jhydene.2010.05.092

[16] A. Rokhmanenkov, A. Yanilkin. Int. J. Hydrogen Energy, 44, 29132 (2019). DOI: 10.1016/j.ijhydene.2019.03.237

[17] T.L. Murashkina, M.S. Syrtanov, R.S. Laptev, A.M. Lider. Int. J. Hydrogen Energy, 44, 6709 (2019). DOI: $10.1016 /$ j.jihydene.2019.01.150

[18] N.I. Rodchenkova, Yu.V. Zaika. Int. J. Hydrogen Energy, 36 (1), 1239 (2011). DOI: 10.1016/j.ijhydene.2010.06.121

[19] Yu.V. Zaika, E.K. Kostikova. Adv. Mater. Sci. Appl., 3 (3), 120 (2014). DOI: 10.5963/AMSA0303003

[20] Yu.V. Zaika, E.K. Kostikova. Int. J. Hydrogen Energy, 42 (1), 4005 (2017). DOI: 10.1016/j.jhydene.2016.10.104

[21] E. Legrand, A. Oudriss, C. Savall, J. Bouhattate, X. Feaugas. Int. J. Hydrogen Energy, 40 (6), 2871 (2015).

DOI: 10.1016/j.ijhydene.2014.12.069
[22] Yu.S. Nechaev, N.M. Alexandrova, N.A. Shurygina, A.O. Cheretaeva, E.K. Kostikova, A. Ochsner. J. Nucl. Mater. 535, 152162 (2020). DOI: 10.1016/j.jnucmat.2020.152162

[23] Yu.S. Nechaev, N.M. Alexandrova, O.S. Cheretaeva, V.L. Kuznetsov, A. Öchner, E.K. Kostikova, Yu.V. Zaika. Int. J. Hydrogen Energy, 45, 25030 (2020). DOI: $10.1016 /$ j.jihydene.2020.06.242

[24] П. Шьюмон. Дифбузия в твердых телах. (Металлургия, M., 1966) [Пер. с англ.: P.G. Shewmon. Diffusion in solids, (McGraw-Hill Book Company, Inc., NY-San FranciscoToronto-London)]

[25] H. Atsumi, Y. Kondo. Fusion Eng. Des., 131, 49 (2018). DOI: 10.1016/j.fusengdes.2018.04

[26] H. Atsumi, Y. Takemura, T. Miyabe, T. Konishi, T. Tanabe, T. Shikama. J. Nucl. Mater. 442, S746 (2013). DOI: 10.1016/j.jnucmat.2013.03.041 